\title{
Correction to: Real-World Tumor Response of Palbociclib Plus Letrozole Versus Letrozole for Metastatic Breast Cancer in US Clinical Practice
}

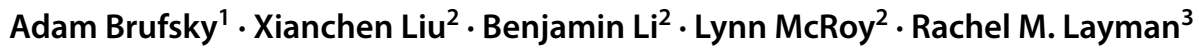

Published online: 15 October 2021

(c) Springer Nature Switzerland AG 2021

\section{Correction to: Targeted Oncology (2021) 16: 601-611 https://doi.org/10.1007/s11523-021-00826-1}

A peer-reviewed Plain Language Summary was retrospectively added to this publication.

\section{Plain Language Summary}

Palbociclib (Ibrance $囚 ;$ PAL) is a treatment for advanced or metastatic breast cancer (MBC for short). Metastatic means that the cancer has spread from breast tissue to other areas of the body. PAL is taken with medications known as hormone therapy, such as letrozole (LET), to treat people with a type of MBC called hormone receptor-positive, human epidermal growth factor receptor 2-negative (HR+/ HER2-). Researchers wanted to understand more about how treatment with PAL+LET affected tumor response (ie, if the tumor shrinks or disappears) in women with $\mathrm{HR}+/$ HER2- MBC. We looked at tumor response to PAL+LET in a real-life setting using anonymous medical record information from a database of about 2.4 million US patients with cancer. This study included 1383 patients with HR+/HER2MBC who started PAL+LET (754 patients) or LET alone (629 patients) as their first MBC treatment from 2015-2018. Among people who took PAL+LET, $60 \%$ people had a

The original article can be found online at https://doi.org/10.1007/ s11523-021-00826-1.

Adam Brufsky

brufskyam@upmc.edu

$\triangle$ Xianchen Liu

Jasonxc.Liu@pfizer.com

1 College of Medicine, University of Pittsburgh, 300 Halket St., Suite 4628, Pittsburgh, PA 15213, USA

2 Pfizer Inc, New York, NY, USA

3 The University of Texas MD Anderson Cancer Center, Houston, TX, USA tumor response compared with $39 \%$ of people who took LET alone. Of the people who took PAL+LET, half of the people lived with their cancer without it getting worse for at least 20 months compared with 15 months for people who took LET alone. These results add to what is known about PAL+LET treatment based on routine clinical practice and clinical trials. Both types of information provide support for PAL+LET treatment as the standard care for women with HR+/HER2- MBC.

Palbociclib is approved to treat the condition under study that is discussed in this summary.

This summary reports the results of a single study. The results of this study may differ from those of other studies. Health professionals should make treatment decisions based on all available evidence not on the results of a single study.

This study described is still ongoing, therefore the final outcomes of this study may differ from the outcomes described in this summary.

Funding This study was sponsored by Pfizer (ClinicalTrials.gov identifier: NCT04176354).

Acknowledgements Writing support was provided by Lauriaselle Afanador, PhD, of ICON plc (North Wales, PA) and was funded by Pfizer.

The original article has been updated. 